Supporting Information for:

\title{
The Importance of Unbound Ligand in Nanocrystal Superlattice Formation
}

\author{
Samuel W. Winslow ${ }^{\dagger}$, James W. Swan ${ }^{*}$, , and William A. Tisdale ${ }^{*}, \dagger$ \\ ${ }^{\dagger}$ Department of Chemical Engineering, Massachusetts Institute of Technology, Cambridge, \\ Massachusetts 02142, United States
}

\section{S.1. Experimental Details}

S.2. GISAXS Unit Cell Measurements

S.3. Molecular Dynamics Simulation Ligand Distributions.

S.4. Molecular Dynamics Simulation Shell Thickness Fitting.

S.5. Model of Spin-Coating 


\section{S.1. Experimental Details.}

1) Synthesis and Batch Characterization. PbS NCs were synthesized according to a previously published hot-injection method with $\mathrm{PbCl}_{2}$ (Alfa Aesar, 99.999\%) and elemental S (SigmaAldrich, $\geq 99.99 \%$ ) in oleylamine (Sigma-Aldrich, $\geq 98 \%$ primary amine) as the precursors. ${ }^{1} \mathrm{~A}$ $\mathrm{Pb} / \mathrm{S}$ ratio of 24:1 was used. The NCs were precipitated by the addition of $10 \mathrm{~mL}$ 1-butanol (SigmaAldrich, $\geq 99.4 \%$ ) and $5 \mathrm{~mL}$ methanol (Sigma-Aldrich, $\geq 99.9 \%$ ) to each $30 \mathrm{~mL}$ portion of the synthesized NC dispersion. The NCs were redispersed in $15 \mathrm{~mL}$ hexanes (Sigma-Aldrich, $\geq$ $98.5 \%$ ) and $200 \%$ by volume oleic acid (Sigma-Aldrich, $90 \%$ ) was added. The NCs were agitated and then precipitated by centrifugation at $4300 \mathrm{rpm}$ for 3 minutes. The supernatant was an orange color, demonstrating unreacted sulfur precursor remained in solution. This redispersion with hexanes and precipitation with oleic acid was repeated until the supernatant was clear $(3-4$ repetitions). A final precipitation by addition of $\sim 10 \mathrm{~mL}$ 1-butanol $\sim 5 \mathrm{~mL}$ methanol to the NCs dispersed in $20 \mathrm{~mL}$ of hexanes was performed to remove excess ligand from the solution. There is a change in solution turbidity denoting the decreased NC solubility. This step is relatively uncontrolled and is the origin of small quantities of unbound ligand remaining in solution. Additional crashouts with alcohols were performed to strip ligands from the surface and lower the ligand coverage.

Absorption spectra were measured using a Cary 5000 UV-vis-NIR spectrophotometer. For measurement, the NCs were suspended in tetrachloroethylene. Core diameters and concentrations were measured using a previously published sizing curve. ${ }^{1,2}$ The three varied ligand coverages used in the controlled additions did not show a measureable shift in core size as the first excitonic peak in their absorption spectra remained unchanged after successive alcohol crashouts and removal of ligands from the surface (Figure S1). 


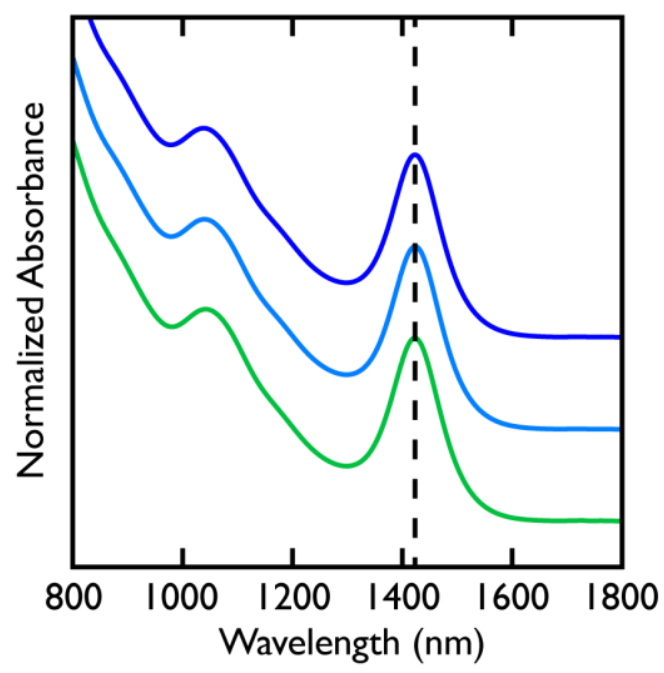

Figure S1. Normalized absorption spectra (offset) for the varied ligand coverage samples used in the controlled additions. There is no difference in the absorption peak positions $(1423 \mathrm{~nm})$ for the NCs with 6.2 ligands $/ \mathrm{nm}^{2}$ (green), 5.2 ligands $/ \mathrm{nm}^{2}$ (light blue), and 4.1 ligands $/ \mathrm{nm}^{2}$ (dark blue).

2) Proton Nuclear Magnetic Resonance Spectroscopy. ${ }^{1} \mathrm{H}-\mathrm{NMR}$ was performed on a threechannel Bruker Avance Neo spectrometer operating at $500.34 \mathrm{MHz}$ equipped with a $5 \mathrm{~mm}$ liquidnitrogen cooled Prodigy broad band observe (BBO) cryoprobe. The instrument runs in fullautomation off of a SampleXpress 60 sample charger. Known quantities of NCs, measured by absorbance, ${ }^{2}$ were dissolved in $650 \mu \mathrm{L}$ of deuterated toluene (Sigma-Aldrich, $\geq 99.6 \% \mathrm{C}_{7} \mathrm{D}_{8}$ ) containing a known quantity $(2 \mu \mathrm{L})$ of dibromomethane as an internal quantitative standard. Measurement temperature was $25^{\circ} \mathrm{C}$. The spectra were measured under conditions of full $\mathrm{T}_{1}$ relaxation (delay of $45 \mathrm{~s})$. The integrated area under the $\mathrm{CH}_{2} \mathrm{Br}_{2}(\sim 4 \mathrm{ppm})$, bound ligand vinyl proton $(5.7 \mathrm{ppm})$, and free ligand vinyl proton $(5.5 \mathrm{ppm})$ resonances were used to measure the number of ligands per NC. The bound and free ligand vinyl proton resonances are well separated so that the populations can be separately quantified. 
3) X-ray Scattering. NC thin film samples were prepared by spin-coating on $18 \times 18 \mathrm{~mm}$ glass wafers. Prior to use, the substrates were cleaned and treated overnight with a $0.02 \mathrm{M}$ (3mercaptopropyl)trimethoxysilane (MPTS) solution in toluene to improve NC adhesion. For all samples except where noted otherwise, $100 \mu \mathrm{L}$ of a $50 \mathrm{mg} / \mathrm{mL}$ suspension of NCs in toluene were pipetted onto the substrate. The samples were then spun at $1500 \mathrm{rpm}$ for $30 \mathrm{~s}$ in a vented chamber. Initial GISAXS patterns were collected at the D1 beamline of the Cornell High Energy Synchrotron Source (CHESS). The X-ray beam was produced by a hardbent dipole magnet and a Mo: $\mathrm{B} 4 \mathrm{C}$ multilayer double-bound monochromator, with the radiation having a wavelength of $1.164 \AA$ at a bandwidth of $1.5 \%$. The GISAXS patterns were collected on a DECTRIS Pilatus3 $200 \mathrm{~K}$ detector. The sample-to-detector distances were calibrated using a silver behenate standard. An incident angle of $0.3^{\circ}$ with respect to the X-ray beam was used. A $0.1 \mathrm{~s}$ exposure time was used.

GISAXS patterns for the controlled additions of oleic acid were collected on the SAXSLAB system in the MIT Materials Research Laboratory (MRL) X-ray Diffraction Shared Experimental Facility. The system uses a Rigaku 002 microfocus X-ray source with an Osmic staggered parabolic multilayer optic system to focus the beam crossover at the second pinhole. Two sets of JJ X-ray 4 jaw collimation slits are used. The X-ray wavelength was $1.538 \AA$. Samples are measured in a vacuum chamber that is pumped down to 0.08 mbar. Patterns were collected on a Pilatus3 300K detector at a sample-to-detector distance of $1059 \mathrm{~mm}$ using an incident angle of $0.3^{\circ}$ and an integration time of $30 \mathrm{~min}$.

GISAXS patterns were indexed with in-house software ${ }^{3,4}$ which allows for selection of lattice type, lattice parameters, and superlattice plane parallel to the substrate. The software overlays expected scattering peaks onto the patterns. The patterns were indexed manually by eye to ensure 
agreement between high intensity peaks at low scattering vectors as well as higher-order reflections.

3) Molecular Dynamics Simulations. PbS NCs are faceted in shape with exposed $\{100\}$ and $\{111\}$ crystal planes. By specifying the number of atoms along any adjoined planes, we can generate core models in the typical truncation sequence from octahedron, through truncated octahedron, to cuboctahedron. Here we use shapes close to a regular truncated octahedron. Ligands were grafted exclusively onto (111) facets in line with computational and experimental results. ${ }^{5-7}$ Coverage was manually varied as ligand coverage is known to vary in experiment. The number of free ligands in the simulation was also varied. Electrostatic interactions were excluded from the simulations. The core was prescribed as a rigid body and ligands were bound to individual lead atoms with a stiff harmonic bond such that no surface rearrangement was allowed. No solvent was used. The united atom representation was used to coarse grain $\mathrm{CH}_{\mathrm{x}}$ groups as individual beads. The following potentials were used: ${ }^{8}$

$$
\begin{gathered}
\text { Harmonic Bond }\left(\mathrm{sp}^{3} \mathrm{sp}^{3}\right): U(r)=\frac{k_{\text {bond }}}{2}\left(r-r_{0}\right)^{2} \\
\text { Harmonic Bond }\left(\mathrm{sp}^{3} \mathrm{sp}^{2}\right): U(r)=\frac{3 k_{\text {bond }}}{4}\left(r-r_{0}\right)^{2} \\
\text { Harmonic Bond }\left(\mathrm{sp}^{2} \mathrm{sp}^{2}\right): U(r)=k_{\text {bond }}\left(r-r_{0}\right)^{2} \\
\text { Harmonic Angle: } U(\theta)=\frac{k_{\text {angle }}}{2}\left(\cos \theta-\cos \theta_{0}\right)^{2} \\
\text { Dihedral Angle }\left(\mathrm{sp}^{3} \mathrm{sp}^{3}\right): U(\varphi)=\sum_{n=1}^{3} k_{n}\left(1+(-1)^{n+1} \cos n \varphi\right) \\
\text { Dihedral Angle }\left(\mathrm{sp}^{3} \mathrm{sp}^{2}\right): U(\varphi)=c_{0}+\sum_{n=1}^{3} c_{n}\left(1+(-1)^{n+1} \cos n \varphi\right) \\
\text { Dihedral Angle }\left(\mathrm{sp}^{2} \mathrm{sp}^{2}\right): U(\varphi)=\frac{k_{\mathrm{phi}}}{2}(\varphi)^{2}
\end{gathered}
$$


Here, $r$ is the distance between two beads, $\theta$ is the angle between three beads, and $\varphi$ is the dihedral angle. We use the convention that a dihedral angle of 0 corresponds to a cis configuration. Table S1 contains the intramolecular parameters used. 
Table S1. Ligand potential parameters.

\begin{tabular}{cccc}
\hline Potential Type & Parameter & Value & Units \\
\hline \multirow{2}{*}{ Bonds } & $k_{\text {bond }}$ & 634 & $\mathrm{kcal} /\left(\mathrm{mol} / \AA^{2}\right)$ \\
& $r_{0}$ & 1.53 & $\AA$ \\
\hline \multirow{2}{*}{ Angles } & $k_{\text {angle }}$ & 120 & $\mathrm{kcal} / \mathrm{mol}$ \\
& $\theta_{0}$ & 1.92 & $\mathrm{radian}$ \\
\hline \multirow{5}{*}{ Dihedral Angles } & $k_{1}$ & 355.03 & $\mathrm{~K}$ \\
& $k_{2}$ & -68.19 & $\mathrm{~K}$ \\
& $k_{3}$ & 791.32 & $\mathrm{~K}$ \\
& $c_{0}$ & 688.5 & $\mathrm{~K}$ \\
& $c_{1}$ & 86.36 & $\mathrm{~K}$ \\
& $c_{2}$ & -109.77 & $\mathrm{~K}$ \\
& $c_{3}$ & -282.24 & $\mathrm{~K}$ \\
& $k_{\text {phi }}$ & 24800 & $\mathrm{~K}$ \\
\hline
\end{tabular}

Parameters describing Lennard-Jones type interactions of the core with ligands were modeled after simulations of PbSe NCs with the same interaction energy and distance for lead, while the parameters for sulfur were back-calculated using Lorentz-Berthelot mixing rules. ${ }^{8-10}$ Table S2 summarizes these parameters.

Table S2. MD simulation interaction parameters.

\begin{tabular}{cccc}
\hline Molecule & Atom/Group & $\boldsymbol{\epsilon}(\mathbf{k c a l} / \mathbf{m o l})$ & $\boldsymbol{\sigma}(\AA)$ \\
\hline \multirow{2}{*}{$\mathrm{PbS} \mathrm{NC} \mathrm{Core}$} & $\mathrm{Pb}$ & 0.0596 & 3.29 \\
& $\mathrm{~S}$ & 0.1051 & 4.22 \\
\hline \multirow{3}{*}{ Ligands } & $\mathrm{CH}_{3}\left(\mathrm{sp}^{3}\right)$ & 0.22644 & 4.009 \\
& $\mathrm{CH}_{2}\left(\mathrm{sp}^{3}\right)$ & 0.09344 & 4.009 \\
& $\mathrm{CH}\left(\mathrm{sp}^{2}\right)$ & 0.10032 & 3.695 \\
\hline
\end{tabular}

Simulations were run in the NVT ensemble using the HOOMD-blue software package ${ }^{11-13}$ with mass, length, and energy scales of $1 \mathrm{u}, 1 \AA$, and $1 \mathrm{~kJ} / \mathrm{mol}$. A time step of $5 \mathrm{fs}$ was used. A large enough simulation box was used so that the NC did not interact with itself beyond the $10 \AA$ 
interaction cutoff distance. Simulations were run long enough to see the ligand-shell thickness approach a value that did not change with increased time. 


\section{S.2. GISAXS Unit Cell Measurements}

1) Variability in Initial GISAXS Samples. Disagreement between the unit cell volume when only accounting for core and bound ligands motivated the examination of unbound ligand, as described in the main text. The agreement between predicted and measured unit cell volumes when accounting for both unbound and bound ligand highlighted the need to account for the unbound ligand when predicting SL structure. The parameters for the data presented in Figure 1c are summarized in Table . The core sizes, bound coverage, and free ligand fractions span the typical ranges.

Table S3. Experimental parameters for the data presented in Figure 1c. Core diameter $D$ and dispersity $\sigma_{d}$ are measured using the peak position and peak width of the first excitonic feature in the absorbance spectra. Dispersity is given as a percentage of the core diameter. Ligand coverage $\gamma$ and free ligand fraction $\phi_{\mathrm{f}}$ are measured using ${ }^{1} \mathrm{H}-\mathrm{NMR}$. Unit cell axes are measured by GISAXS.

\begin{tabular}{cccccc}
\hline $\boldsymbol{D}(\mathbf{n m})$ & $\boldsymbol{\sigma}_{\boldsymbol{d}}(\mathbf{\%})$ & $\boldsymbol{\gamma}\left(\mathbf{l i g} / \mathbf{n m}^{\mathbf{2}}\right)$ & $\boldsymbol{\phi}_{\mathbf{f}}(\mathbf{l i g} / \mathbf{N C})$ & $\boldsymbol{a}, \boldsymbol{b}(\mathbf{n m})$ & $\boldsymbol{c}(\mathbf{n m})$ \\
\hline 4.69 & 5.8 & 3.07 & 0.65 & 11.0 & 11.0 \\
4.97 & 5.6 & 3.35 & 0.72 & 12.6 & 12.6 \\
5.28 & 3.9 & 4.16 & 0.55 & 12.55 & 11.4 \\
5.46 & 4.3 & 5.82 & 0.50 & 13.25 & 13.8 \\
5.55 & 5.1 & 4.41 & 0.47 & 13.45 & 12.15 \\
5.75 & 4.2 & 5.33 & 0.46 & 13.2 & 13.2 \\
6.22 & 4.4 & 3.93 & 0.55 & 14.18 & 12.85 \\
6.61 & 4.1 & 4.57 & 0.40 & 14.6 & 14.6 \\
6.85 & 4.1 & 4.39 & 0.44 & 14.6 & 14.6 \\
6.93 & 4.0 & 4.92 & 0.12 & 15.15 & 14.6 \\
6.98 & 4.8 & 4.87 & 0.05 & 14.6 & 11.4 \\
\hline
\end{tabular}


2). Phase Space Comparison. The initial variability in observed SL structures spanned the phase space from BCC to FCC. For comparison to the SL tuning from BCC to FCC observed during the controlled additions, in Figure S2 we plot the other SL structures in the phase space of Figure 2b. It is expected that each additional sample could be tuned across the phase space through the manipulation of bound and unbound ligand.

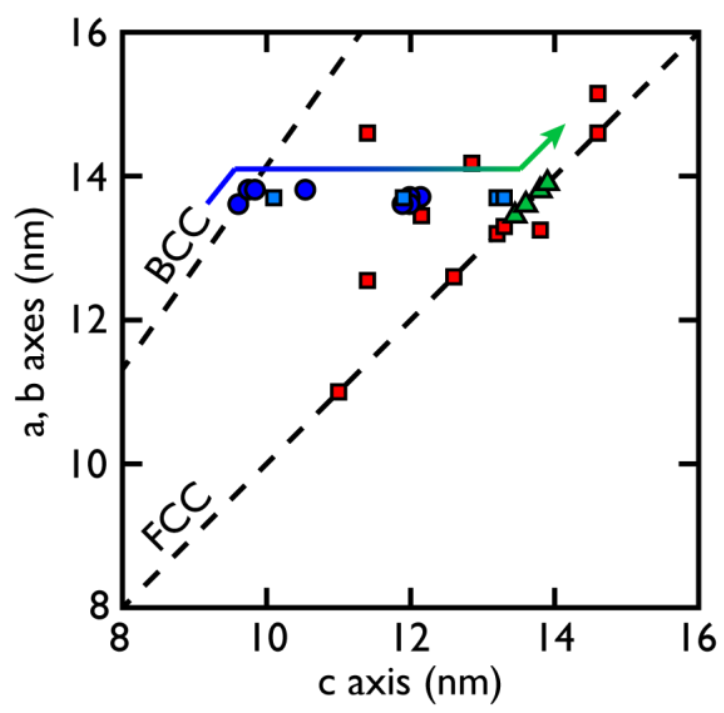

Figure S2. Comparison of the controlled additions tuning pathway to the initial variability in observed SLs. Adding free ligand shifts each batch with bound coverage of 4.1 ligands $/ \mathrm{nm}^{2}$ (dark blue circles), 5.2 ligands $/ \mathrm{nm}^{2}$ (light blue squares), and 6.2 ligands $/ \mathrm{nm}^{2}$ (green triangles) towards FCC. The additional samples (red squares) with lattice parameters in Table have SL structures throughout the phase space.

3). Unit Cell Evolution for Controlled Additions. A selection of GISAXS patterns is presented in Figure 2a in the main text though more samples are plotted in Figure 2b. Table contains the complete unit cell descriptions for all the controlled addition samples. 
Table S4. Complete experimental parameters for the controlled additions. Core diameter $D$ and dispersity $\sigma_{d}$ are measured using the peak position and peak width of the first excitonic feature in the absorbance spectra. Dispersity is given as a percentage of the core diameter. Ligand coverage $\gamma$ and free ligand fraction $\phi_{\mathrm{f}}$ are measured using ${ }^{1} \mathrm{H}-\mathrm{NMR}$. Unit cell axes are measured by GISAXS.

\begin{tabular}{cccccc}
\hline $\boldsymbol{D}(\mathbf{n m})$ & $\boldsymbol{\sigma}_{\boldsymbol{d}} \mathbf{( \% )}$ & $\boldsymbol{\gamma}\left(\mathbf{l i g} / \mathbf{n m}^{\mathbf{2}}\right)$ & $\boldsymbol{\phi}_{\mathbf{f}}(\mathbf{l i g} / \mathbf{N C})$ & $\boldsymbol{a}, \boldsymbol{b}(\mathbf{n m})$ & $\boldsymbol{c}(\mathbf{n m})$ \\
\hline 6.1 & 3.4 & 4.1 & 0 & 13.6 & 9.62 \\
6.1 & 3.4 & 4.1 & 0.10 & 13.8 & 9.76 \\
6.1 & 3.4 & 4.1 & 0.25 & 13.8 & 9.85 \\
6.1 & 3.4 & 4.1 & 0.50 & 13.8 & 10.55 \\
6.1 & 3.4 & 4.1 & 0.75 & 13.7 & 12.15 \\
6.1 & 3.4 & 4.1 & 0.80 & 13.7 & 12 \\
6.1 & 3.4 & 4.1 & 0.84 & 13.6 & 12 \\
6.1 & 3.4 & 4.1 & 0.88 & 13.6 & 12 \\
6.1 & 3.4 & 4.1 & 0.90 & 13.6 & 11.9 \\
\hline 6.1 & 3.4 & 5.2 & 0 & 13.7 & 10.1 \\
6.1 & 3.4 & 5.2 & 0.25 & 13.7 & 11.9 \\
6.1 & 3.4 & 5.2 & 0.50 & 13.7 & 13.2 \\
6.1 & 3.4 & 5.2 & 0.80 & 13.7 & 13.3 \\
\hline 6.1 & 3.4 & 6.2 & 0.12 & 13.45 & 13.45 \\
6.1 & 3.4 & 6.2 & 0.25 & 13.6 & 13.6 \\
6.1 & 3.4 & 6.2 & 0.50 & 13.8 & 13.8 \\
6.1 & 3.4 & 6.2 & 0.80 & 13.9 & 13.9 \\
\hline
\end{tabular}




\section{S.3. Molecular Dynamics Simulation Ligand Distributions}

Free ligand readily associates with the bound ligand-shell. Figure S3 shows distributions of the core (black), bound ligand (cyan), and free ligand (red) densities relative to their bulk value for various core sizes, coverages, and free ligand fractions. These plots are analogous to Figure $3 \mathrm{~b}$ of the main text. Within a panel, the bound ligand coverage is fixed and free ligand fraction increases. Across each row, the bound ligand coverage increases from 2.3 to 5.8 ligands $/ \mathrm{nm}^{2}$ at a fixed $\mathrm{NC}$ core diameter. Down each column, the NC core diameter increases at fixed ligand coverage. Universally, the peak in the free ligand distributions increase and move farther out in the ligandshell as free ligand fraction increases. The ligand presence over each $\mathrm{NC}$ facet is proportional to the NC surface area of each facet. For the highest ligand coverage (rightmost column), the bound ligand distribution does not change significantly with free ligand fraction because the ligand-shell is already completely extended. There is minimal room to expand farther. The shift outwards of the ligand distributions is consistent with the notion of transitioning $\mathrm{BCC} \rightarrow \mathrm{FCC}$ and increasing the nearest-neighbor spacing. It also suggests the $\mathrm{NC}$ interactions must become more isotropic in nature as the core and ligand coverage anisotropy is masked by a larger ligand-shell. 

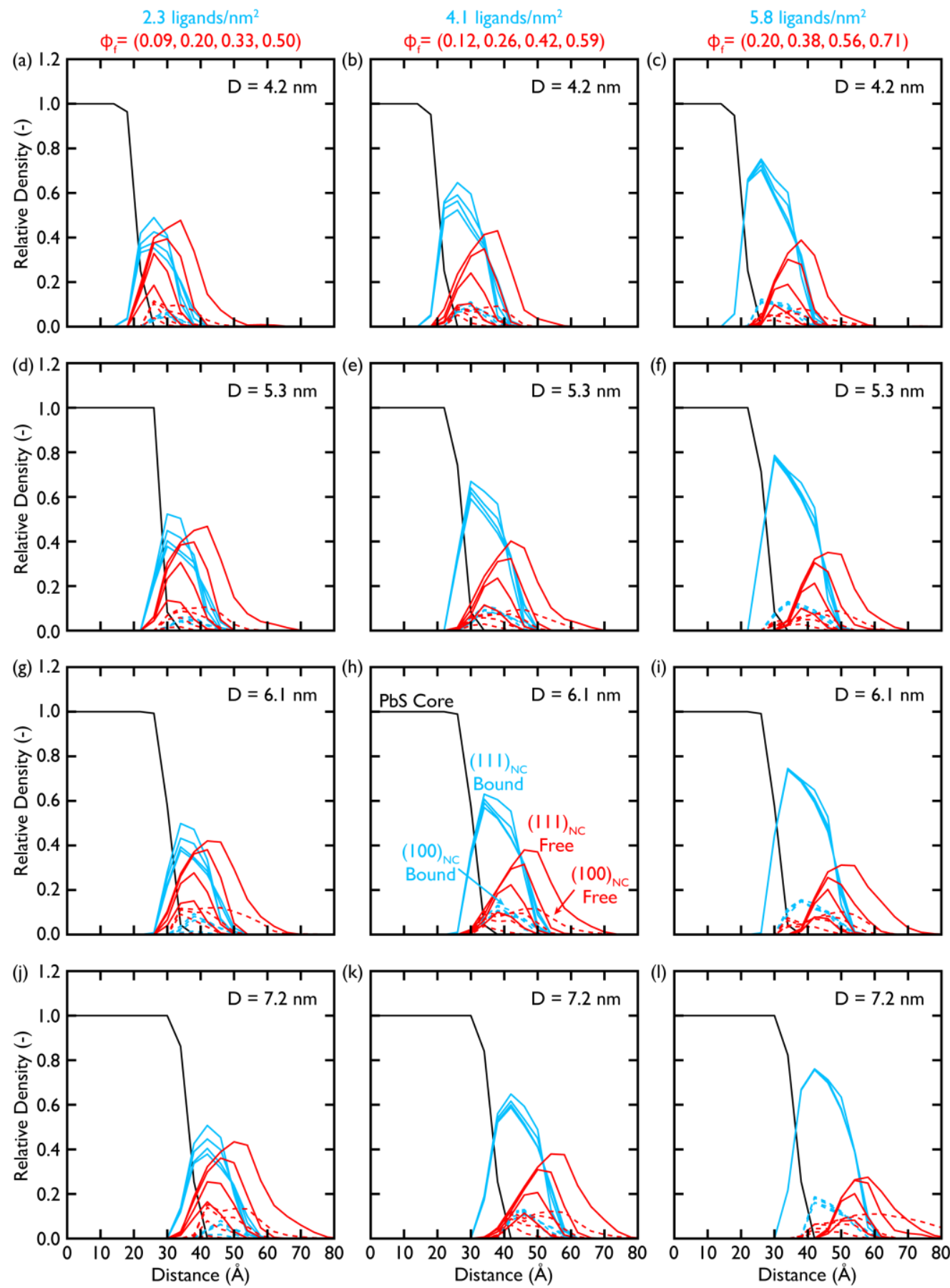

Figure S3. Relative density distributions of bound (cyan) and free (red) ligands which are located over the $(111)_{\mathrm{NC}}$ facets (solid) and $(100)_{\mathrm{NC}}$ facets (dashed). Across each row, ligand coverage increases from 2.3 to 5.8 ligands $/ \mathrm{nm}^{2}$ at fixed $\mathrm{NC}$ core diameter. Down each column the $\mathrm{NC}$ diameter increases at fixed ligand coverage. Free ligand fractions for each column are noted. Figure $3 \mathrm{~b}$ from the main text is identical to panel (h). 


\section{S.4. Molecular Dynamics Simulation Shell Thickness Fitting}

1) Model Derivation. As shown in Figure 3c, when the ligand shell thickness extracted from MD simulation is plotted as a function of the total number of ligands (bound and unbound), the data for a given core size collapse onto single trends. This motivated fitting to a soft-max function where the shell thickness increases initially linearly from a minimum, levelling out to a maximum limit as the number of ligands increases with an adjustable sharpness of the turnover. The softmax function has the form:

$$
t\left(N_{\text {lig }} ; D, \gamma, \phi_{\mathrm{f}}\right)=t_{0}+\left(t_{\max }-t_{0}\right) \frac{N_{\text {lig }}\left(D, \gamma, \phi_{\mathrm{f}}\right)}{\left(N_{\max }(D)^{p}+N_{l i g}\left(D, \gamma, \phi_{\mathrm{f}}\right)^{p}\right)^{1 / p}}
$$

where $t$ is the predicted shell thickness, $D$ is the core diameter, $\gamma$ is the ligand coverage, $\phi_{\mathrm{f}}$ is the free ligand fraction, $t_{0}$ is the minimum relaxed length of a bound ligand, $t_{\text {max }}$ is the length of a fully extended bound ligand, $N_{\text {lig }}$ is the total number of ligands, $N_{\max }$ is the number of ligands to occupy a volume defined by the shell thickness, and $p$ is an exponent to be fit defining the sharpness of the turnover in the soft-max function. This function is parameterized by the measurable experimental parameters that characterize a specific batch of NCs.

The minimum shell thickness is taken from the polymer literature as $t_{0}=a g^{3 / 5}$ where $a$ is the bead size and $g$ is the number of beads. Using a bead size of $1.53 \AA$ from the simulation parameters and 18 beads in a chain, we obtain a minimum shell thickness of $8.67 \AA$. Visually this matches the data well. The value for $t_{\max }$ is taken as the distance across the terminal ligands beads in the chain when at its maximal extended conformation plus the surface bond length. This yields a value for $t_{\max }$ of $21.9 \AA$. These values could have been fit to the data. However, as reasonable estimates exist, we use those values to reduce the number of fit parameters. 
For a maximal shell thickness $t_{\max }$ on a NC with core size $D$, there is a fixed number of ligands that can occupy the available volume in the ligand shell. Thus, $N_{\max }$ is found for a given core size as:

$$
N_{\max }(D)=\frac{4 \pi \psi}{3 V_{m}}\left(\left(\frac{D}{2}+t_{\max }\right)^{3}-\left(\frac{D}{2}\right)^{3}\right) .
$$

Here, $V_{m}$ is the ligand molecular volume taken to be $0.524 \mathrm{~nm}^{3} /$ ligand. The core sphericity $\psi$ is included as the spherical shell volume surrounding a faceted NC core is smaller than the equivalent spherical shell volume around a spherical NC core. We use a sphericity of 0.92 , which is the maximum for a $\mathrm{NC}$ core in the truncated octahedron family.

The number of ligands in the simulation is the sum of the bound and unbound ligands:

$$
N_{\text {lig }}\left(D, \gamma, \phi_{\mathrm{f}}\right)=N_{\text {bound }}(D, \gamma)+N_{\text {unbound }}\left(D, \gamma, \phi_{\mathrm{f}}\right) .
$$

The number of bound ligands is calculated from the core size and ligand coverage as:

$$
N_{\text {bound }}(D, \gamma)=\frac{\pi D^{2} \gamma}{\psi} .
$$

Here too, the sphericity is included to account for the increased surface area of the NC core compared to that of a sphere. Importantly, we define the number of unbound ligands as the number of ligands that associate with the bound ligand shell. It is irrelevant if extra ligands are floating around far from the NC. To calculate this association, we assume a Langmuir type adsorption so that the fractional occupation of available sites for adsorption is controlled by the partial pressure or concentration of free ligands in the simulation. A Langmuir isotherm would be written as:

$$
\frac{N_{\text {unbound }}}{N_{\text {sites }}}=\frac{K C_{\text {unbound }}}{1+K C_{\text {unbound }}},
$$

where $N_{\text {sites }}$ is the number of available sites for adsorption, $K$ is a parameter describing the strength of adsorption, and $C_{\text {unbound }}$ represents the concentration of unbound ligands. This partial 
pressure of unbound ligands is precisely the free ligand fraction $\phi_{\mathrm{f}}$. We will rewrite the adsorption isotherm as:

$$
N_{\text {unbound }}\left(D, \gamma, \phi_{\mathrm{f}}\right)=\frac{1}{\frac{K^{\prime}}{\phi_{\mathrm{f}}}+1} N_{\text {sites }}(D, \gamma),
$$

with a new fit parameter $K^{\prime}$ as the inverse of $K$. We must now find a functional form for the number of available sites $N_{\text {sites }}$ which depends on the core size and ligand coverage. This is simply taken as the maximal number of ligands that could fit in the ligand shell at full extension minus the number of bound ligands that are already in the shell:

$$
N_{\text {sites }}(D, \gamma)=N_{\text {max }}(D)-N_{\text {bound }}(D, \gamma) .
$$

The final expression for the $N_{\text {lig }}$ then takes the form:

$$
N_{l i g}\left(D, \gamma, \phi_{\mathrm{f}}\right)=\frac{\pi D^{2} \gamma}{\psi}+\frac{N_{\max }(D)-\frac{\pi D^{2} \gamma}{\psi}}{\frac{K^{\prime}}{\phi_{\mathrm{f}}}+1} .
$$

The fitting function gives the predicted shell thickness given the experimental measurables of core size $D$, ligand coverage $\gamma$, and free ligand fraction $\phi_{\mathrm{f}}$ with two fit parameters $p$ and $K^{\prime}$. The fit parameters are found by simultaneously fitting all the MD simulation data, with values found to be 4.4 and 0.5 respectively. 


\section{S.5. Model of Spin-Coating}

1) Governing Equations. The modeling of spin-coating in the main text follows the approach of Meyerhofer, the implementation of which follows. ${ }^{14}$ The total thin film height is taken to be the sum of the solvent/liquid $(L)$ and the solute/solid/NC $(S)$. These quantities vary in time such that the time varying film height is defined as:

$$
h(t)=S+L .
$$

The concentration is given as the fraction of film height attributable to the solids:

$$
c(t)=\frac{S}{S+L} .
$$

The governing differential equations for spin-coating are given by:

$$
\begin{gathered}
\frac{d S}{d t}=-c(t) \frac{2 \omega^{2} h(t)^{3}}{3 v(t)} \\
\frac{d L}{d t}=-(1-c(t)) \frac{2 \omega^{2} h(t)^{3}}{3 v(t)}-E,
\end{gathered}
$$

Where $\omega$ is the spin speed, $v(t)=f(c(t))$ is the kinematic viscosity which depends on the concentration and thus time, and $E$ is a constant evaporation term. Solids are only lost by convection of the solution off the edge of the substrate. Liquid is lost due to both convection and evaporation. We adopt functional forms identical to Meyerhofer for the concentration-dependent viscosity and constant solvent evaporative terms as:

$$
\begin{gathered}
v(t)=v_{\text {toluene }}+v_{\text {oleic acid }} c(t)^{r}, \\
E=k \omega^{1 / 2} .
\end{gathered}
$$

We take values for $r$ and $k$ as 2 and $6.32 * 10^{-9}$ respectively, similar to Meyerhofer. These could ultimately be tuned to match measured film heights and drying times, but we find decent agreement in estimated drying times of 10-11 s from watching spun-cast sample preparations. The equations are solved using an explicit Euler method with time step of $1 \mathrm{~ms}$ and an initial film height of 31 
$\mu \mathrm{m}$, consistent with the $100 \mu \mathrm{L}$ dropcast volume over the $18 \times 18 \mathrm{~mm}$ substrate surface area. For the three free ligand fractions presented in the main text, our model would predict film heights of 58,82 , and $175 \mathrm{~nm}$ for increasing free ligand fractions. These are in reasonable agreement with previous measured film heights of approximately $120 \mathrm{~nm} .{ }^{15}$ As such, we take the qualitative behavior of the model as accurate using the viscosity scaling and evaporation rate parameters state above. 


\section{References}

(1) Weidman, M. C.; Beck, M. E.; Hoffman, R. S.; Prins, F.; Tisdale, W. A. Monodisperse, AirStable PbS Nanocrystals via Precursor Stoichiometry Control. ACS Nano 2014, 8 (6), 63636371.

(2) Moreels, I.; Lambert, K.; Smeets, D.; De Muynck, D.; Nollet, T.; Martins, J. C.; Vanhaecke, F.; Vantomme, A.; Delerue, C.; Allan, G.; Hens, Z. Size-Dependent Optical Properties of Colloidal PbS Quantum Dots. ACS Nano 2009, 3 (10), 3023-3030.

(3) Busch, P.; Rauscher, M.; Smilgies, D.-M.; Posselt, D.; Papadakis, C. M. Grazing-Incidence Small-Angle X-Ray Scattering from Thin Polymer Films with Lamellar Structures - the Scattering Cross Section in the Distorted-Wave Born Approximation. J. Appl. Crystallogr. 2006, $39(3), 433-442$.

(4) Smilgies, D.-M.; Blasini, D. R. Indexation Scheme for Oriented Molecular Thin Films Studied with Grazing-Incidence Reciprocal-Space Mapping. J. Appl. Crystallogr. 2007, 40 (4), 716-718.

(5) Choi, J. J.; Bealing, C. R.; Bian, K.; Hughes, K. J.; Zhang, W.; Smilgies, D.-M.; Hennig, R. G.; Engstrom, J. R.; Hanrath, T. Controlling Nanocrystal Superlattice Symmetry and ShapeAnisotropic Interactions through Variable Ligand Surface Coverage. J. Am. Chem. Soc. 2011, $133(9), 3131-3138$.

(6) Drijvers, E.; De Roo, J.; Martins, J. C.; Infante, I.; Hens, Z. Ligand Displacement Exposes Binding Site Heterogeneity on CdSe Nanocrystal Surfaces. Chem. Mater. 2018, 30 (3), $1178-1186$. 
(7) Kessler, M. L.; Dempsey, J. L. Mapping the Topology of PbS Nanocrystals through Displacement Isotherms of Surface-Bound Metal Oleate Complexes. Chem. Mater. 2020, $32(6), 2561-2571$.

(8) Kaushik, A. P.; Clancy, P. Solvent-Driven Symmetry of Self-Assembled Nanocrystal Superlattices - A Computational Study. J. Comput. Chem. 2013, 34 (7), 523-532.

(9) Schapotschnikow, P.; Van Huis, M. A.; Zandbergen, H. W.; Vanmaekelbergh, D.; Vlugt, T. J. H. Morphological Transformations and Fusion of PbSe Nanocrystals Studied Using Atomistic Simulations. Nano Lett. 2010, 10 (10), 3966-3971.

(10) Grünwald, M.; Zayak, A.; Neaton, J. B.; Geissler, P. L.; Rabani, E. Transferable Pair Potentials for CdS and ZnS Crystals. J. Chem. Phys. 2012, 136 (23), 1-6.

(11) Anderson, J. A.; Lorenz, C. D.; Travesset, A. General Purpose Molecular Dynamics Simulations Fully Implemented on Graphics Processing Units. J. Comput. Phys. 2008, 227 (10), 5342-5359.

(12) Glaser, J.; Nguyen, T. D.; Anderson, J. A.; Lui, P.; Spiga, F.; Millan, J. A.; Morse, D. C.; Glotzer, S. C. Strong Scaling of General-Purpose Molecular Dynamics Simulations on GPUs. Comput. Phys. Commun. 2015, 192, 97-107.

(13) Nguyen, T. D.; Phillips, C. L.; Anderson, J. A.; Glotzer, S. C. Rigid Body Constraints Realized in Massively-Parallel Molecular Dynamics on Graphics Processing Units. Comput. Phys. Commun. 2011, 182 (11), 2307-2313.

(14) Meyerhofer, D. Characteristics of Resist Films Produced by Spinning. J. Appl. Phys. 1978, 49 (7), 3993-3997. 
(15) Weidman, M. C.; Yager, K. G.; Tisdale, W. A. Interparticle Spacing and Structural Ordering in Superlattice PbS Nanocrystal Solids Undergoing Ligand Exchange. Chem. Mater. 2014, $27(2), 474-482$. 